\title{
Amplifying Waveguide Optical Isolator With an Integrated Electromagnet
}

\author{
Wouter Van Parys, Student Member, IEEE, Dries Van Thourhout, Member, IEEE, Roel Baets, Fellow, IEEE, \\ Beatrice Dagens, Jean Decobert, Odile Le Gouezigou, Dalila Make, and Liesbet Lagae
}

\begin{abstract}
We have demonstrated an amplifying waveguide optical isolator with an integrated electromagnet. This provides a solution to the generally poor magnetic remanence of this type of isolator. The proof of principle is presented and optimization routes are discussed.
\end{abstract}

Index Terms-Magnetooptic Kerr effect, optical isolators, semiconductor optical amplifiers (SOAs).

\section{INTRODUCTION}

A $\mathrm{N}$ optical isolator is indispensable in an optical telecom link to protect semiconductor lasers from optical feedback. A planar, waveguide version of the optical isolator is highly desirable as it would greatly reduce the packaging cost-hence the overall cost — of a laser diode module, because it avoids the expensive alignment required with current commercial bulk isolators.

An integratable isolator configuration that is getting a lot of attention in recent years is the amplifying waveguide optical isolator [1]-[4]. The device basically is a semiconductor optical amplifier (SOA) with a transversally magnetized ferromagnetic film close to the guiding core. The magnetooptic (MO) Kerr effect causes a nonreciprocal shift of the complex effective index of the guided modes, implying that the modal loss is dependent on the propagation direction. Electrical biasing decreases the overall loss level in the device. The result is a device which, being transparent in one direction while providing loss in the opposite direction, is isolating. The main advantage of this isolator concept is that monolithic integration with a semiconductor laser is straightforward, as both components are essentially the same. Configurations operating in transverse-magnetic (TM) polarization [1], [3], [4] and transverse-electric (TE) polarization [2] have been demonstrated.

One of the main issues for this kind of device is the magnetization of the ferromagnetic metal film. The very high aspect ratio of the length of the film to the width results in very low remanent magnetization of the film due to large demagnetizing fields. One of the solutions to this problem is to have a

Manuscript received July 23, 2007; revised September 6, 2007. This research has been carried out in the framework of the IST-ISOLASER Project.

W. Van Parys, D. Van Thourhout, and R. Baets are with the Photonics Research Group, Department of Information Technology (INTEC), Ghent University-IMEC, B-9000 Gent, Belgium (e-mail: Wouter.VanParys@ intec.UGent. be).

B. Dagens, J. Decobert, O. Le Gouezigou, and D. Make are with Alcatel Thales III-V Lab, 91767 Palaiseau, France.

L. Lagae is with IMEC vzw, B-3001 Leuven, Belgium.

Color versions of one or more of the figures in this letter are available online at http://ieeexplore.ieee.org.

Digital Object Identifier 10.1109/LPT.2007.909634 magnet integrated with the isolator. In this letter, we demonstrate a TM-mode amplifying waveguide isolator with an integrated electromagnet. This magnet is a gold strip deposited along the longitudinal direction of the isolator in close vicinity to the ferromagnetic film, which in this configuration both acts as the source of the nonreciprocity and as the electrical contact for the SOA. Current flowing through the gold strip from one side of the cavity to the other generates a TM field causing the MO Kerr effect.

Apart from the application as an optical isolator, this result is interesting in itself as it is now possible to modify the internal loss of an SOA dynamically and nonreciprocally.

\section{DESIGN}

The design of the amplifying waveguide optical isolator and the integrated electromagnet can be considered as two separate parts. The design of the isolator itself was extensively described in our previous work [4], hence we will limit ourselves here to a description of the component. The amplifying core is an $\mathrm{AlGaInAs}\left(\lambda_{g}=1288 \mathrm{~nm}\right)$ tensile-strained $(-1.6 \%$ strain) multiple quantum-well structure, where the built-in tensile strain provides TM-selective material gain while suppressing TE-gain. Among the studied ferromagnetic metals $\mathrm{Co}_{50} \mathrm{Fe}_{50}$ was found to be the best compromise between a high MO effect and low absorption [5]. Low electrical resistivity of the metal-semiconductor contact combined with minor additional absorption is provided by a hybrid $\mathrm{Be} \mathrm{p}^{+}$-doped $\mathrm{In}_{0.81} \mathrm{Ga}_{0.19} \mathrm{As}_{0.41} \mathrm{P}_{0.59}-\mathrm{In}_{0.54} \mathrm{Ga}_{0.46}$ As bilayer [6]. Proper design of the thickness of the top cladding layer and the two separate confinement heterostructure layers gives the optimized theoretical performance; $62 \mathrm{~mA}$ suffices to achieve transparency on a 2-mm-long isolator providing 10-dB optical isolation [7], where the ridge width was set at $2 \mu \mathrm{m}$.

A schematic cross section of the amplifying waveguide optical isolator with integrated electromagnet is given in the top part of Fig. 1. The design parameters for the electromagnet are the width of the gold stripe and the distance between the electromagnet and the ferromagnetic metal film. Other parameters are the thickness of the gold stripe, which influences the current density in the electromagnet but not the strength of the magnetic field, and the variation of the generated magnetic field in the lateral direction ( $x$-direction), which is of only minor importance if the stripe width is much larger than the width of the ferromagnetic film. The latter has to be the case for the isolator to ensure that the magnetic field is aligned along the lateral direction ( $x$-direction).

The partial differential equation describing the magnetic field problem was solved numerically. The bottom part of Fig. 1 shows the result of these calculations. The simulated values of the magnetic field (in Oe) are plotted for variation of 


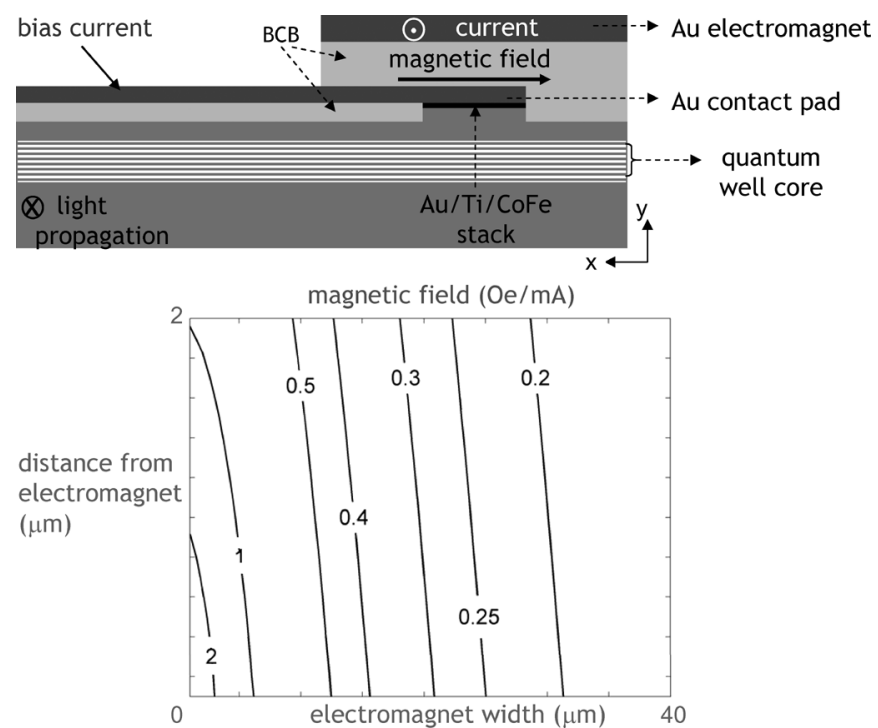

Fig. 1. (top) Schematic cross section of the TM-mode amplifying waveguide isolator with integrated electromagnet. (bottom) Simulation of the lateral magnetic field ( $x$-direction) as a function of the width of the electromagnet gold stripe and the distance ( $y$-direction) from the electromagnet, expressed in Oe per milliampere of current injection.

the width of the electromagnet and the distance from the electromagnet per unit (milliampere) current injection in the gold stripe. For sufficiently wide stripes the generated magnetic field is quasi-independent of the distance from the electromagnet. This is an advantage for the fabrication because the use of a thick separation layer between bias contact and electromagnet reduces the risk of short-circuiting both metal layers.

With the available lithography mask it was only possible to have an electromagnet gold stripe as wide as $30 \mu \mathrm{m}$, resulting in a magnetic field that is far from optimized. However, it is suitable for a proof-of-principle experiment [8].

\section{FABRICATION}

The isolator was grown with metal-organic vapor phase epitaxy on an $\mathrm{n}^{+}$S-doped InP substrate $\left(\mathrm{N}_{S}=3.0 \times 10^{18} \mathrm{~cm}^{-3}\right)$. The layer structure is a $\mathrm{Si}$-doped $\left(\mathrm{N}_{\mathrm{Si}}=10^{18} \mathrm{~cm}^{-3}\right)$ InP cladding layer, the nonintentionally doped (n.i.d.) optimized active core, a Be p-doped InP cladding $\left(\mathrm{N}_{\mathrm{Be}}=\right.$ $\left.5.0 \times 10^{17} \mathrm{~cm}^{-3} \rightarrow 2.0 \times 10^{18} \mathrm{~cm}^{-3}\right)$ and the optimized contact structure. The sputter-deposited 50-nm-thick $\mathrm{Co}_{50} \mathrm{Fe}_{50}$ film, capped with a $40-\mathrm{nm} / 150-\mathrm{nm} \mathrm{Ti}-\mathrm{Au}$ protective bilayer, was patterned into $3-\mu \mathrm{m}$-wide stripes with standard lift-off. Ridge waveguides were defined with $\mathrm{CH}_{4}: \mathrm{H}_{2}$ plasma reactive ion etching using these metallic stripes as an etch mask. With this technique, full covering of the ridge with metal has been achieved. An additional 30- $\mu \mathrm{m}$-wide gold stripe is evaporated to enable contacting of the isolator, separated from the semiconductor surface by a benzocyclobutene (BCB) current isolation layer. On top of the isolator bias contact a $30-\mu \mathrm{m}$-wide and 215-nm-thick gold stripe is deposited, serving as the electromagnet. Both gold stripes are separated by a $1-\mu \mathrm{m}$-thick $\mathrm{BCB}$ layer. Isolators with a cavity length of $1.3 \mathrm{~mm}$ have been cleaved and mounted for characterization.

In Fig. 2, two scanning electron microscope (SEM) images of a cross section of the amplifying waveguide optical isolator with integrated electromagnet are given. The left picture shows

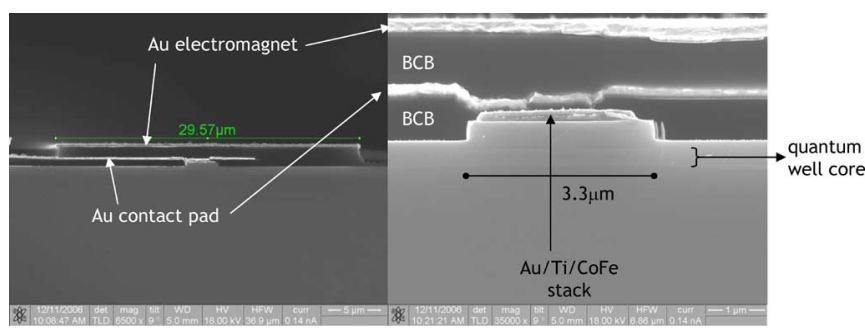

Fig. 2. SEM pictures of the cross section of the amplifying waveguide optical isolator with integrated electromagnet.

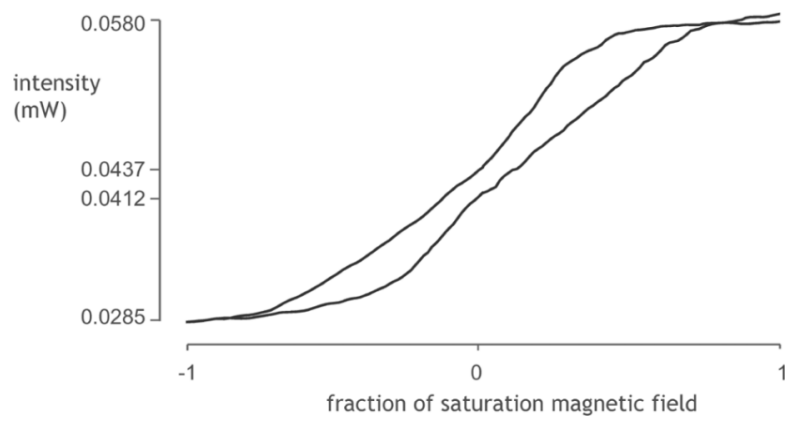

Fig. 3. Optical power emitted by the isolator as a function of the externally applied magnetic field at a bias current of $140 \mathrm{~mA}$ (= above threshold).

that the fabricated device is in agreement with the design. When zooming in on the ridge waveguide (right part of Fig. 2), it can be seen that the definition of the ridge is not perfect, with a ridge width that is slightly wider than designed and roughness present at the side of the ridge. While this has definitely its influence on the isolator performance, these inaccuracies are not expected to change the operation of the integrated electromagnet.

\section{CHARACTERIZATION}

An easy and fast characterization method for the amplifying waveguide optical isolator is to pump as-cleaved isolators above threshold and extract the emitted power as a function of the applied lateral magnetic field-switching the magnetic field direction is equivalent to switching the light propagation direction. Because the material gain is clamped above threshold, the nonreciprocal loss shift is independent of the isolator bias current and the optical isolation can be extracted from the ratio of forward to backward intensity-or equivalently the ratio of the intensity at magnetization in either lateral direction

$$
\text { isolation }[\mathrm{dB}]=2 \frac{10}{\ln 10} \ln (\text { intensity ratio). }
$$

This method is used throughout this letter. The amplifying waveguide isolator was initially characterized with an external electromagnet as a reference for the integrated electromagnet. The laser threshold current equals $103 \mathrm{~mA}$ and the lasing wavelength is $1288 \mathrm{~nm}$. In Fig. 3, the emitted power is plotted as a function of the externally applied magnetic field for a bias current of $140 \mathrm{~mA}$ on a $1.3-\mathrm{mm}$-long isolator. The corresponding maximal isolation, at saturation magnetization, is $6.2 \mathrm{~dB}$. This graph shows that the remanent isolation-at zero magnetic field-is only $8.3 \%$ of the maximum value.

Next, the integrated electromagnet was characterized by applying current to the gold strip while pumping the isolator above threshold. To avoid heating, the electromagnet is driven with 

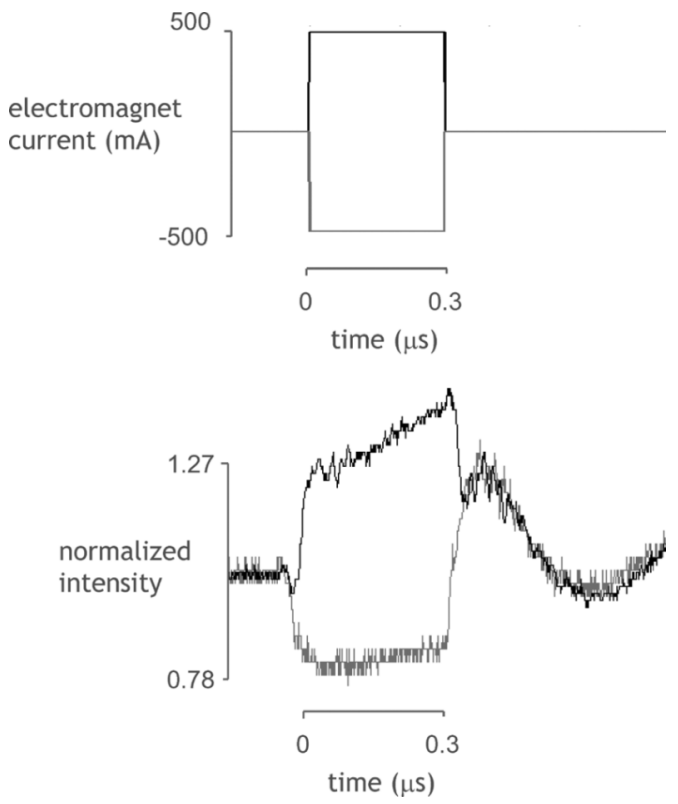

Fig. 4. (top) Time evolution of the injected (pulsed) current in the electromagnet. (bottom) Corresponding (normalized) intensity emitted by the optical isolator at a bias current of $140 \mathrm{~mA}$ (= above threshold).

pulsed current (pulsewidth $0.3 \mu$ s, duty cycle $3 \%$ ). The chip temperature is controlled with a thermoelectric cooler at a value of $20{ }^{\circ} \mathrm{C}$. The isolator bias current is continuous wave. The output signal is evaluated on a digital sampling oscilloscope. The measurement is done for both directions of the current flow in the electromagnet, corresponding to a magnetic field in either lateral direction. The bottom part of Fig. 4 shows the measured time evolution of the intensity. The corresponding pulsed current is plotted in the top part of Fig. 4, where negative current corresponds to reversed current flow direction. The intensity is normalized to compensate for variations in the fiber-to-chip coupling. At the on-set of the current pulse the emitted power rapidly increases or decreases, depending on the current flow direction. This is a clear proof of the on-set of the nonreciprocal loss shift. After the off-set of the pulse, the emitted power quickly becomes identical again in both cases and then slowly recovers from the heating of the device. The ratio of the intensity at "forward" to that at "backward" current injection equals 1.64 , which for an isolator of 1.3-mm cavity length corresponds to an isolation of $4.3 \mathrm{~dB}$, or $70 \%$ of what is achieved at saturation magnetization.

We repeated the measurements for different values of the electromagnet current injection. In Fig. 5, the corresponding evolution of the optical isolation with injected (pulsed) current is plotted (bottom axis). For comparison, the optical isolation as a function of the strength of an externally applied magnetic field is also given (top axis). Fitting both graphs shows that with a current injection of $500 \mathrm{~mA}$, the achieved magnetic field is half the value needed to saturate the ferromagnetic film.

The results in Fig. 5 shows that a very high current of $1.1 \mathrm{~A}$ is needed to achieve magnetic saturation with the fabricated electromagnet. However, as previously mentioned, due to limitations in the fabrication, the demonstrated electromagnet design is far from optimized. Decreasing the width of the gold strip to $5 \mu \mathrm{m}$ will decrease the required current by a factor of 5 . In order not to increase the current density—hence the dissipated

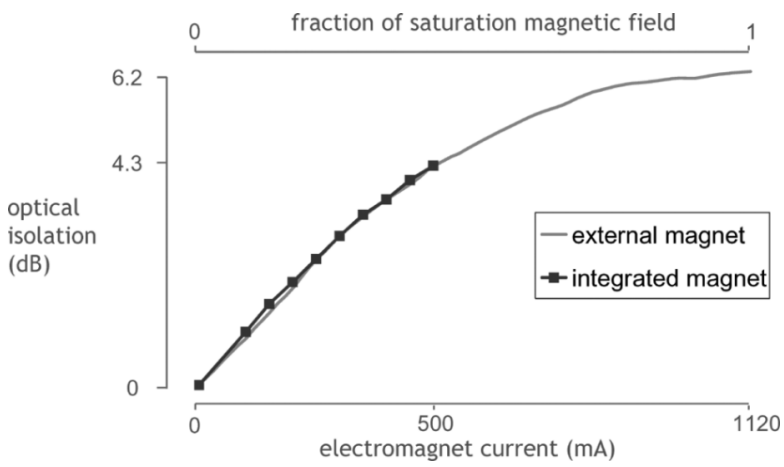

Fig. 5. Evolution of the optical isolation with current in the electromagnet (bottom axis) and with externally applied magnetic field (top axis).

heat - the thickness of the gold film must accordingly be increased by a factor of 6 . This has only a minor influence on the generated magnetic field. Furthermore, modification of the ferromagnetic film might decrease the magnetic field-hence the current level—required to saturate the film, a point which needs further investigation.

\section{CONCLUSION}

We have developed an optical isolator with an integrated electromagnet. While the demonstrated drive current is beyond the acceptable level, possibilities for major improvement have been identified. Further research is however required, as the electromagnet drive current should be lower than $50 \mathrm{~mA}$ to limit its contribution to the overall power consumption of the amplifying waveguide optical isolator.

\section{REFERENCES}

[1] M. Vanwolleghem, W. Van Parys, D. Van Thourhout, R. Baets, F. Lelarge, O. Gauthier-Lafaye, B. Thedrez, R. Wirix-Speetjens, and L. Lagae, "Experimental demonstration of nonreciprocal amplified spontaneous emission in a CoFe clad semiconductor optical amplifier for use as an integrated optical isolator," Appl. Phys. Lett., vol. 85, pp. 3980-3982, 2004.

[2] H. Shimizu and Y. Nakano, "Fabrication and characterization of an InGaAsP/InP active waveguide optical isolator with $14.7 \mathrm{~dB} / \mathrm{mm} \mathrm{TE}$ mode nonreciprocal attenuation," J. Lightw. Technol., vol. 24, no. 1, pp. 38-43, Jan. 2006.

[3] V. Zayets and K. Ando, "Isolation effect in ferromagnetic-metal/semiconductor hybrid optical waveguide," Appl. Phys. Lett., vol. 86, pp. 261105-261107, 2005.

[4] W. Van Parys, B. Moeyersoon, D. Van Thourhout, R. Baets, M. Vanwolleghem, B. Dagens, J. Decobert, O. Le Gouezigou, D. Make, R. Vanheertum, and L. Lagae, "Transverse magnetic mode nonreciprocal propagation in an amplifying AlGaInAs/InP optical waveguide isolator,' Appl. Phys. Lett., vol. 88, p. 071115, 2006.

[5] M. Vanwolleghem, P. Gogol, P. Beauvillain, W. Van Parys, and R. Baets, "Design and optimization of a monolithically integratable InPbased optical waveguide isolator," J. Opt. Soc. Amer. B, vol. 24, pp. 94-105, 2007.

[6] W. Van Parys, D. Van Thourhout, R. Baets, M. Vanwolleghem, F. Lelarge, B. Thedrez, and L. Lagae, "Study of a magnetooptic contact for an amplifying waveguide optical isolator," IEEE Photon. Technol. Lett., vol. 19, no. 9, pp. 659-661, Sep. 2007.

[7] W. Van Parys, D. Van Thourhout, R. Baets, B. Dagens, J. Decobert, O. Le Gouezigou, D. Make, R. Vanheertum, and L. Lagae, "11.4 dB isolation on an amplifying AlGaInAs/InP optical waveguide isolator," presented at the OFC 2006, U.S., 2006, Paper OFA2.

[8] W. Van Parys, D. Van Thourhout, R. Baets, B. Dagens, J. Decobert, O. Le Gouezigou, D. Make, and L. Lagae, "Amplifying waveguide optical isolator with integrated electromagnet," presented at the ECIO 2007, Denmark, 2007, Paper ThA5. 